\title{
Validating a Density Functional Theory Approach for Predicting the Redox Potentials Associated with Charge Carriers and Excitons in Polymeric Photocatalysts
}

\author{
Pierre Guiglion, Adriano Monti, and Martijn A. Zwijnenburg*(i) \\ Department of Chemistry, University College London, 20 Gordon Street, London WC1H 0AJ, U.K.
}

Supporting Information

ABSTRACT: We compare, for a range of conjugated polymers relevant to water-splitting photocatalysis, the predictions for the redox potentials associated with charge carriers and excitons by a totalenergy $\triangle \mathrm{DFT}$ approach to those measured experimentally. For solidstate potentials, of the different classes of potentials available experimentally for conjugated polymers, the class measured under conditions which are the most similar to those during water splitting, we find a good fit between the ionization potentials predicted using $\triangle \mathrm{B} 3 \mathrm{LYP}$ and those measured experimentally using photoemission spectroscopy (PES). We also observe a reasonable fit to the more limited data sets of excited-state ionization potentials, obtained from two-photon PES, and electron affinities, measured by inverse PES, respectively. Through a comparison of solid-state potentials with gas

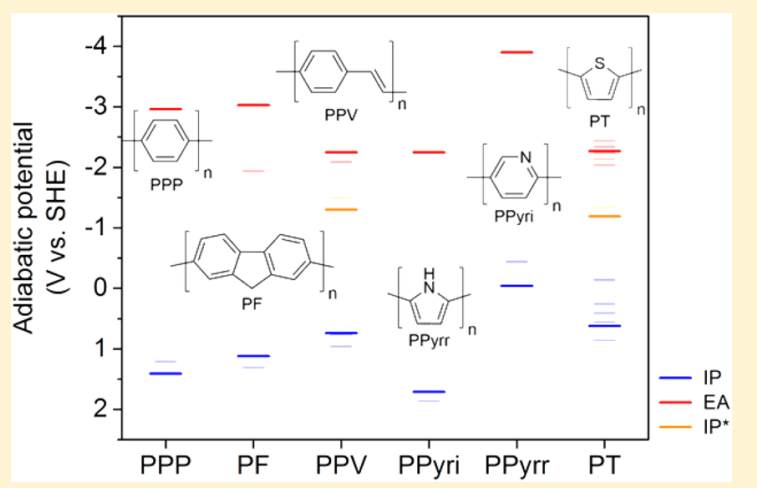
phase and solution potentials for a range of oligomers, we demonstrate how the quality of the fit to experimental solid-state data is probably the result of benign error cancellation. We discuss that the good fit for solid-state potentials in vacuum suggests that a similar accuracy can be expected for calculations on solid-state polymers interfaced with water. We also analyze the quality of approximating the $\triangle B 3 L Y P$ potentials by orbital energies. Finally, we discuss what a comparison between experimental and predicted potentials teaches us about conjugated polymers as photocatalysts, focusing specifically on the large exciton-binding energy in these systems and the mechanism of free charge carrier generation.

\section{INTRODUCTION}

Oligomeric and polymeric photocatalysts ${ }^{1-13}$ that can drive the redox half-reactions that underlie photocatalytic water splitting, i.e., the reduction of protons to molecular hydrogen and/or oxidation of water to oxygen gas, or $\mathrm{CO}_{2}$ photoreduction, are currently a very active area of research. ${ }^{14,15}$ The attraction of these materials arises from the fact that they are based on earth abundant elements, that their properties can easily be tuned through copolymerization, and that, compared to related carbon nitride ${ }^{16-27}$ and graphene oxide ${ }^{28,29}$ photocatalysts, their molecular structure is generally well understood.

One of the crucial requirements for a potential photocatalyst, polymeric or not, to meet is the thermodynamic ability to drive both of the water splitting or $\mathrm{CO}_{2}$ photoreduction solution half-reactions. This prerequisite, at least for colloidal photocatalysts, translates into the constraint that the adiabatic potentials associated with the charge carriers, the ionization potential (IP), and electron affinity (EA) for holes and electrons, respectively, and the excited state, IP* and EA*, in a materials straddle those of the solution half-reactions, as shown in Figure 1. Knowledge of these potentials is hence crucial when trying to understand the activity of known photocatalysts or developing new ones. Reliably measuring such potentials, especially under operating conditions, however, is far from easy. For example, cyclic voltammetry $(\mathrm{CV})$ on solid polymer films on an electrode in contact with an aprotic polar solvent system, the closest measurements in practice come to a solid polymer in contact with water, is generally hard to interpret because the voltammograms tend to be broad, show signs of irreversibility, ${ }^{30-32}$ and involve the incorporation of counterions and solvent molecules into the film, ${ }^{33}$ something not expected under photocatalysis conditions. A related problem with $\mathrm{CV}$ and other electrochemical measurements is that measuring the electron affinity values of polymers is complicated by the fact that these often lie outside the stability window of common solvents and that one thus has to be cautious not to confuse a signal due to solvent oxidation with the signature of electron affinity. The ability to calculate the redox potentials of charge carriers and excitons, therefore, is very useful, especially as it moreover allows one to predict such potentials of hypothetical materials that have not been synthesized as yet and thus to screen computationally for promising photocatalysts. The latter is an especially attractive proposition for polymeric materials, for which the synthesis,

Received: November 5, 2016

Revised: December 22, 2016

Published: December 22, 2016 


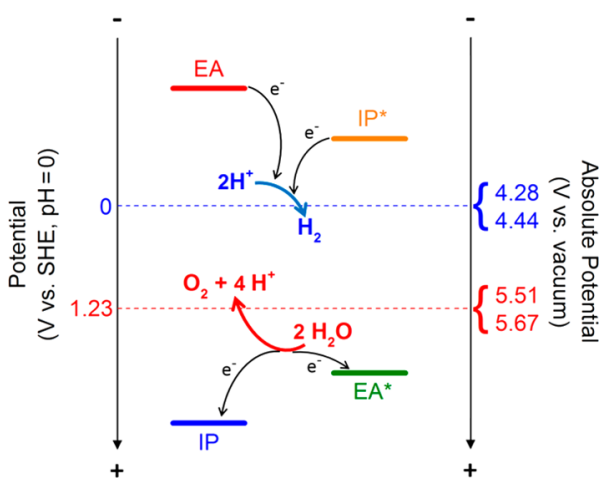

Figure 1. Scheme illustrating how, in the case of water splitting, the redox potentials associated with charge carriers (IP and EA) and excitons $\left(\mathrm{EA}^{*}\right.$ and $\left.\mathrm{IP}^{*}\right)$ of a photocatalyst must straddle the proton reduction and water oxidation potentials (blue and red broken lines, respectively) for both processes to be thermodynamically favorable. The vertical axis on the right of the figure shows the different possible alignments with vacuum, the result of the different experimental SHEAP values.

purification, and characterization of both the polymer and constituting monomers can be very time-consuming.

In our group, we developed a density functional theory (DFT) approach specifically aimed at predicting the potentials of polymeric and nanoparticulate photocatalysts ${ }^{34-38}$ and applied it, among other things, to linear polymers, ${ }^{11,34}$ including the first ever reported polymer photocatalyst poly ( $p$-phenylene), ${ }^{1-4}$ polymer networks, ${ }^{8}$ and carbon nitride materials. ${ }^{35}$ The main difference between our approach and that developed by others ${ }^{39-45}$ for describing photocatalysts is that it revolves around molecular calculations in combination with a continuum dielectric screening model ${ }^{46}$ to describe the material and its aqueous environment rather than calculations using periodic boundary conditions (PBC). The focus in the case of polymeric materials on a single polymer strand and the assumption that all intermolecular interaction, be it with other polymer strands or water, can be described in terms of an isotropic dielectric response, is related to the fact that such materials are often amorphous or only poorly crystallized. Hence, we do not have good experimental structural models for polymers to use in PBC calculations and, even if we did construct artificially periodic models, they probably would not necessarily be representative for the solid. Another difference between our approach and that of many others is that we calculate the potentials using a total-energy $\triangle \mathrm{DFT}$ approach, rather than equate IP and EA with (generalized) Kohn-Sham-(G)KS-orbital energies, which at least conceptually is problematic, especially for EA, ${ }^{37,47}$ as well as, calculate potentials associated with the exciton. Finally, we also take into account the (self-)trapping of charge carriers and excitons, i.e., the formation of polarons and polaronic excitons, by considering adiabatic rather than vertical potentials.

Our approach predicts potentials that are in line with observed activity of polymeric photocatalysts; e.g., many polymers are predicted to have no thermodynamic driving force for water oxidation, their ionization potential is more negative than the potential associated with the water oxidation half-reaction, while sacrificial electron donor oxidation is predicted to be exergonic. However, we never previously compared the explicit predicted polymer and oligomer potentials with those measured experimentally. Here, we correct this omission and compare predicted potentials, in the absence of data for polymeric solids in contact with water, to those measured from three distinctly different alternative data sets, gas phase photoemission spectroscopy (PES) for oligomers of $\operatorname{poly}(p$-phenylene $),{ }^{48}$ solution electrochemistry data in aprotic polar solvents for oligomers of poly $(p$ phenylene $)^{49}$ and poly(fluorene $)^{50}$ derivatized with solubilizing alkyl chains, and solid-state (inverse) PES data ${ }^{44,48,51-60}$ for a range of conjugated polymer solids in vacuum. The differences between this and previous work validating the use of hybrid DFT for predicting redox potentials in the literature ${ }^{61-73}$ are a combination of (i) our focus on oligomers/polymers rather than small molecules or metal complexes, (ii) our emphasis on potentials in condensed phases, including the solid-state, (iii) the fact that we consider adiabatic rather than vertical potentials, and/or (iv) because we also include excited state potentials in our comparison. We show that, for conjugated oligomers and polymers, (TD-)DFT calculations using the standard B3LYP ${ }^{74,75}$ density functional yield reasonable gas and solution phase potentials and rather consistently good solidstate potentials, the latter in line with previous work for the IP and EA of small molecules. ${ }^{65-67}$ We further demonstrate that these good solid-state potentials appear to be the result of rather benign error cancellation. We discuss that the good fit for solid-state potentials in vacuum suggests that a similar accuracy can be expected for calculations on solid-state polymers interfaced with water. We will also briefly touch upon the merits of the orbital approximation mentioned above, as well as discuss the requirements on a density functional to consistently calculate the potentials associated with charge carriers and excitons in polymeric materials. Finally, we will examine what a comparison of experimental and computationally predicted potentials teaches us about conjugated polymers as water-splitting photocatalysts.

\section{METHODOLOGY}

We calculate the adiabatic IP, EA, IP*, and EA* potentials of a polymer $\mathrm{P}$ in a $\triangle \mathrm{DFT}$ fashion from the Gibbs free energy difference of the following four redox reactions, written, in line with convention, as reductions

$$
\begin{aligned}
& \mathrm{P}^{+}+\mathrm{e}^{-} \rightarrow \mathrm{P} \\
& \mathrm{P}+\mathrm{e}^{-} \rightarrow \mathrm{P}^{-} \\
& \mathrm{P}^{+}+\mathrm{e}^{-} \rightarrow \mathrm{P}^{*} \\
& \mathrm{P}^{*}+\mathrm{e}^{-} \rightarrow \mathrm{P}
\end{aligned}
$$

where $\mathrm{P}^{+}, \mathrm{P}^{-}$, and $\mathrm{P}^{*}$ are the polymer with a hole, excess electron and exciton, respectively. The Gibbs free energy differences $\Delta G_{r}$ are converted to reduction potentials $E$ via

$$
\Delta G_{r}=-n F E
$$

where $F$ is the Faraday constant and $n$ the number of electrons involved in the reaction, typically one. In our calculations, we furthermore equate the Gibbs free energy difference to the total energy difference, neglecting the vibrational, translational and rotational contribution to the free energy. In our previous work on poly(para-phenylene) oligomers, ${ }^{34}$ this was found to be a generally good approximation because of the relative similarity of the structures of $\mathrm{P}^{+}, \mathrm{P}^{-}, \mathrm{P}^{*}$, and $\mathrm{P}$. Effects of solvation and going to condensed phases in general are described using the $\mathrm{COSMO}^{76}$ dielectric screening model, where we generally neglect the outlying charge correction. $\mathrm{P}^{*}$ energies were obtained by a TD-DFT energy minimization in vacuum, 
Table 1. Experimentally Measured and Computationally Predicted Ionization Potentials of Oligomers and Polymers of $p$ Phenylene in Different Environments vs the Standard Hydrogen Electrode (All Values in Volts)

\begin{tabular}{|c|c|c|c|c|c|c|}
\hline & \multicolumn{2}{|c|}{ gas phase } & \multicolumn{2}{|c|}{$\mathrm{DCM}^{b}$} & \multicolumn{2}{|c|}{ solid } \\
\hline & UV-PES $^{a, 48}$ & $\mathrm{~B} 3 \mathrm{LYP}^{a}$ & $\mathrm{CV}^{c, 49}$ & $\mathrm{~B} 3 L Y P^{a} \varepsilon_{\mathrm{r}} 8.93$ & UV-PES $^{a, 48}$ & ${\mathrm{~B} 3 L Y \mathrm{P}^{a}}^{a} \varepsilon_{\mathrm{r}} 2$ \\
\hline 2 & $3.72(3.88)$ & $3.26(3.42)$ & 1.91 & $1.42(1.58)$ & & \\
\hline 3 & $3.36(3.52)$ & $2.71(2.87)$ & 1.78 & $1.22(1.38)$ & $1.66(1.82)$ & $2.09(2.25)$ \\
\hline 4 & & & 1.73 & $1.13(1.29)$ & & \\
\hline 5 & & & 1.69 & $1.09(1.25)$ & & \\
\hline 6 & $2.76(2.92)$ & $2.08(2.24)$ & 1.66 & $1.07(1.23)$ & $1.46(1.62)$ & $1.63(1.79)$ \\
\hline 7 & & & 1.65 & $1.05(1.21)$ & & \\
\hline 8 & & & 1.65 & & & \\
\hline$\infty$ & & & $1.5^{d}$ & $0.9^{d}$ & $1.21 / 1.36^{e}(1.37 / 1.52)$ & $1.45(1.61)^{f}$ \\
\hline
\end{tabular}

${ }^{a}$ Absolute IP vs vacuum converted to SHE scale by a shift of 4.44 and 4.28 (inside parentheses), respectively. ${ }^{b}$ Oligomers with branched iso-alkyl chains at the terminal p-carbon atoms. ${ }^{c}$ Measured in DCM in the presence of $0.2 \mathrm{Mn}$-Bu $\mathrm{NPF}_{6}$ supporting electrolyte against SCE, values converted to SHE scale by application of a shift of +0.244 . ${ }^{d}$ Obtained through linear extrapolation vs $1 / n$. ${ }^{e}$ Value obtained by two different extrapolation methods in the original experimental paper. ${ }^{f}$ Modeled using an oligomer of 12 units.

followed, if needs be, by a single point TD-DFT calculation using COSMO, where all TD-DFT calculations make the Tamm-Dancoff approximation. ${ }^{77}$ All (TD-)DFT calculations, finally, except where otherwise indicated, were performed using the Turbomole 6.6 code $^{78,79}$ the B3LYP functional, the double$\zeta \mathrm{DZP}^{80}$ basis-set, and the $\mathrm{m} 3$ medium integration grid.

Potentials, be them computationally predicted or experimentally measured, are always expressed with respect to a reference, typically vacuum in the case of photoelectron spectroscopy and a reference electrode, for example, the standard hydrogen electrode (SHE), for liquid electrochemistry. A key parameter in the conversion from one potential reference to another is the value of the SHE absolute potential (SHEAP), for which a range of experimental values are proposed, something that is partly related to different possible choices for thermodynamic standard states and partly due to extra-thermodynamic assumptions. ${ }^{81}$ In this paper, we consider two values of the SHEAP (see Figure 1), $4.44 \mathrm{~V}$, the original IUPAC recommended value ${ }^{82}$ we used in our previous work, and, a more recently proposed value of $4.28 \mathrm{~V}$. ${ }^{81}$ We will present results where possible for both SHEAP choices. However, if in the text only one value is mentioned for a given system, this will be based on the use of $4.44 \mathrm{~V}$ for SHEAP.

\section{RESULTS AND DISCUSSION}

IP. When an oligomer or polymer, or more generally a molecule, is taken from the gas phase into a solution, the solid state or a solid in contact with a solution, the IP of the molecule gets reduced and becomes shallower, through two different mechanisms. ${ }^{83,84}$ First, prior to ionization, in solids, where the molecules are densely packed, hybridization raises the energy of the highest energy occupied orbital, from which an electron will get removed upon ionization. Second, after ionization, the dielectric nature of the environment will screen the generated charge by polarization and stabilize the charged species formed energetically. The difference between the gas and condensed IP values is commonly referred to as the polarization energy, even if only part of this difference is the result of dielectric polarization and, in contrast to what the names suggest, it also contains a contribution due to hybridization.

Table 1 shows experimental and DFT predicted values of the IP of oligomers and polymers of $p$-phenylene in the gas phase, in a dichloromethane (DCM) solution, and as a solid. We will first concentrate on the experimental values taken from the literature. $^{48,49}$ While one has to be slightly careful with comparing these experimental potentials as they are measured using two fundamentally different methods, ultraviolet PES (UV-PES) and CV, and because of the need to convert between different potential references, vacuum for UV-PES and the standard calomel electrode (SCE)/SHE and the ferrocene/ ferrocenium redox couple in the case of $\mathrm{CV}$, such a comparison is very insightful.

As expected based on the literature, the gas phase IPs are the deepest, with the solid-state and DCM solution values being 1$2 \mathrm{~V}$ smaller than the corresponding gas phase values. More interestingly, the polarization energies for the different oligomers in the solid state and DCM, which can be extracted from the IP values in Table 1, are very similar. As the dielectric permittivity of the solid-state oligomer/polymer phase is likely to be smaller than that of DCM, especially given the fact that the supporting electrolyte is likely to increase the effective dielectric permittivity of the DCM solution through ion-pair formation, ${ }^{85}$ this suggests that the smaller dielectric contribution to the permittivity and thus screening of the formed charge in the solid state relative to the solution is more than compensated by the larger contribution due to hybridization in the former. On the basis of the numbers, it is hard to extract an exact value for the effect of hybridization, but it is likely to be at least a few tenths of volts. The experimental polarization energy values measured for the oligomers, finally, are similar in magnitude to those measured for organic crystals ${ }^{83,84}$ and appear to decrease with oligomer length.

Moving on to the computational predictions, where we approximate the alkyl side chain by an isopropyl group (see Figure 2), inspection of Table 1 shows that, for the gas and solution phases, $\triangle \mathrm{DFT}$ calculations using the B3LYP functional yield IP values that are $0.5-0.6 \mathrm{~V}$ shallower, less positive, than those measured experimentally. A similar shift was reported by Baik and Friesner when calculating EA values of small molecules in solution using B3LYP. ${ }^{61}$ Calculations, discussed in the Supporting Information (ESI-1), using a slightly higher

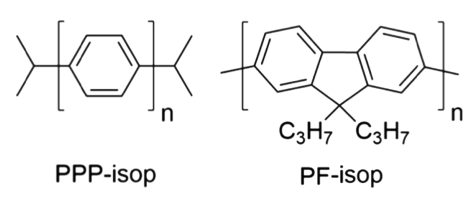

Figure 2. Structures of oligomer models studied computationally. 
dielectric permittivity for the DCM solution than that of pure DCM in order to take into account that the supporting electrolyte will likely increase the effective dielectric permittivity, as well as calculations with larger basis-sets (ESI-2), do not sufficiently change this observation. Moreover, a comparison between similar $\triangle \mathrm{B} 3 \mathrm{LYP}$ calculations and experimental data for the ionization potentials of oligo(fluorene) in solution in the Supporting Information (ESI-1) finds a similar 0.5-0.6 V shift, suggesting this to be a quite general feature. In contrast to for the gas and solution phases, in calculations for the solid state, in which as discussed above we neglect hybridization, the predicted IPs are $0.2-0.4 \mathrm{~V}$ more shallow, less positive, than those measured experimentally. For the dissolved oligomers, the calculations reproduce the experimentally obtained polarization energy values rather well, but the solid-state polarization energies are underestimated by $\sim 1 \mathrm{~V}$. If the isotropic dielectric screening model used in our calculation correctly reproduces the dielectric component of the polarization energy, this would suggest that the effect of hybridization is $\sim 65 \%$ of the polarization energy and far from negligible. The absolute values of the solid-state IPs, most relevant for our work, however, are, as discussed above, reasonably well reproduced. As the inherent density functional related error and the error introduced by neglecting hybridization have similar magnitudes and opposite signs, the solid-state values by benign error cancelation lie close to their experimental counterparts.

This success in reproducing experimental values of solid-state IP values for polymers appears to be not limited to oligomers and the polymer of $p$-phenylene. Figure 3 and Table S5 in the

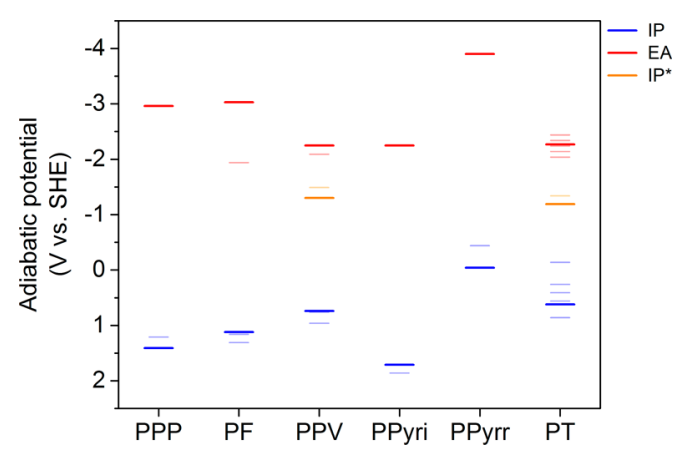

Figure 3. Comparison between the potentials predicted using (TD)B3LYP and $\varepsilon_{\mathrm{r}} 2$, thick lines, and measured experimentally, thin lines, for a range of conjugated polymers.

Supporting Information show a comparison of IPs of a range of conjugated polymers measured experimentally by UV$\mathrm{PES}^{48,51-54,56,60}$ and our B3LYP calculations, again using $\varepsilon_{\mathrm{r}} 2$, for oligomers of 12 units (see Figure 4 for the structures of different polymers studied). Concentrating on the polymers without side chains, for all materials, except perhaps poly(pyrrole), the match is quite good (maximum deviation of $-0.44 \mathrm{~V}$, for poly(pyrrole), and a mean absolute deviation of $0.20 \mathrm{~V}$ ) and the DFT predictions correctly recover the relative ordering of the IPs of the different polymers. Since poly(pyrrole) is easily oxidizable, ${ }^{54}$ the deviation observed for this material can in part find its origin in experiment. Use of a slightly higher dielectric permittivity than 2 for the heteroatom containing polymers, to account for the fact that such polymers probably have a higher dielectric permittivity than pure hydrocarbon polymers (see Table S8 in the Supporting Information), if anything worsens the fit. In this context, it is
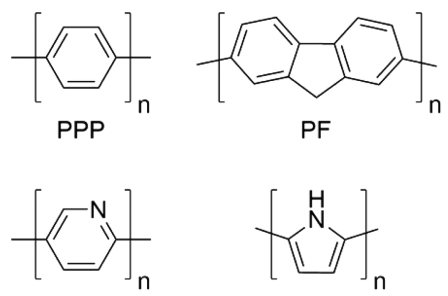

PPyri

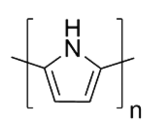

PPyrr
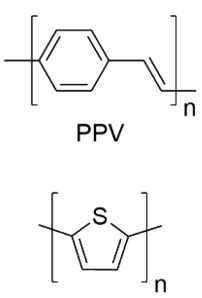

PT
Figure 4. Structures of the polymers studied; the $n$ used in the calculations was 12 for all structures except PF, where an $n$ of 6 was used instead.

important to remember that photoemission is likely to mostly involve molecules near the polymer-vacuum interface due to the surface sensitivity of UV-PES and that such molecules as a result will be less screened than in the bulk. ${ }^{86}$ These results also suggest that the single oligomer embedded in a dielectric continuum approximation, which ignores details of molecular packing, works very well for these amorphous/quasi-crystalline polymers, even if for fully crystalline materials there are cases known where nonisotropic packing effects are large and one has to go beyond the continuum approach. ${ }^{87}$

Overall, on the basis of these results and the fact that the (dielectric) effect of going from an interface with vacuum to water is most likely additive, we feel confident that the computational setup used will also provide decent predictions for the IP of a polymer interfaced with water. Protonation of a polymer might induce an additional shift in the potentials, but this is, even for the nitrogen containing polymers, unlikely to be an issue at (near) neutral $\mathrm{pH}$.

EA. In contrast to the relative multitude of reference data on the IP of oligomers and polymers, there is very little experimental data on the EA of oligomers and polymers, especially in the solid state. Most reported electron affinities are obtained by adding the optical gap, the onset of light absorption, to the value of the IP, but this is a questionable approach. More theoretically justified values can be obtained from either inverse PES or the high kinetic energy edge of twophoton PES (2PPE) spectra. Such data are only available, as far as we are aware, for three of the polymers discussed above. for the alkyl-chain derivatized version of poly(thiophene): poly(3hexylthiophene) (P3HT), ${ }^{59,60}$ poly(fluorene): poly(9,9-dioctylfluorene $)^{57}$ (PF8), and poly(2-methoxy-5-(2-ethyl-hexyloxy)-pphenylene vinylene $)^{58}$ (MEH-PPV). As can be seen from Figure 3 and Table S6 in the Supporting Information, the fit is good for P3HT and PPV, and slightly less good for PF8. This is for calculations neglecting the alkyl side chains. However, calculations that take these side chains into account, included in Table S6 in the Supporting Information, show that these side chains are predicted to only have a small effect on the electron affinity. While these three data points are not sufficient to properly test our approach in terms of its ability to correctly predict electron affinities of polymers in the solid state, it at least give some confidence in our approach. Similarly as for the IP, we expect that the difference in the EA for a polymer in contact with vacuum and water is adequately described by changing the dielectric permittivity from 2 to that of water.

Excited State Potentials: IP* and EA*. Experimental benchmark data for the excited state potentials of relevant polymers are as rare as data for EA, if not rarer. We are aware of 2PPE data, where the process of ionization of the intermediate state by the second photon can be hypothesized to correspond 
to the ionization of a (self-trapped) exciton and thus IP*, for only two polymers: P3HT, ${ }^{59}$ and MEH-PPV. ${ }^{58}$ As can be seen from Figure 3 and Table S7 in the Supporting Information, the experimentally measured values appear in line with the values predicted by our computational approach for the polymers without side chains. Moreover, for both P3HT and MEH-PPV, EA and $\mathrm{IP}^{*}$ are split by $\sim 0.7(\mathrm{e}) \mathrm{V}$, the adiabatic excitonbinding energy, in experiment and by $\sim 1$ (e) $\mathrm{V}$ in our calculations. Similarly as for EA, while these two data points for IP* are not sufficient to properly test our approach in terms of its ability to correctly predict electron affinities of polymers in the solid state, it does give confidence in our approach. We are not aware of any experimental data that would allow us to directly validate our EA* predictions; this would require a 2PPE equivalent inverse photoemission spectroscopy. However, as by definition, the splitting between IP and EA* is the same as between EA and IP*, the fit between predicted IP* and $2 \mathrm{PPE}$ values in combination with the fit between experimental and computational IP values suggests that EA* might be as well described as IP*. Again, just as for IP and EA, the effect of water is likely to be additive for IP* and EA*.

Orbital Approximation. IP and EA are often approximated in the literature by the negative of the energy of the highest energy occupied (G)KS molecular orbital (HOMO or valence band maximum) and lowest energy unoccupied (G)KS molecular orbital (LUMO or conduction band minimum). This approximation is especially problematic on conceptual grounds for EA as, at least for pure density dependent functionals, the unoccupied orbitals are too strongly bound and lie too deep. $^{37,47}$ The (G)KS gap, the energy difference between LUMO and HOMO, as a result lies often closer to the vertical optical gap than the vertical fundamental gap. A comparison between IP/EA and - HOMO/-LUMO for the polymers in Figure 5, however, shows a surprisingly decent fit between both

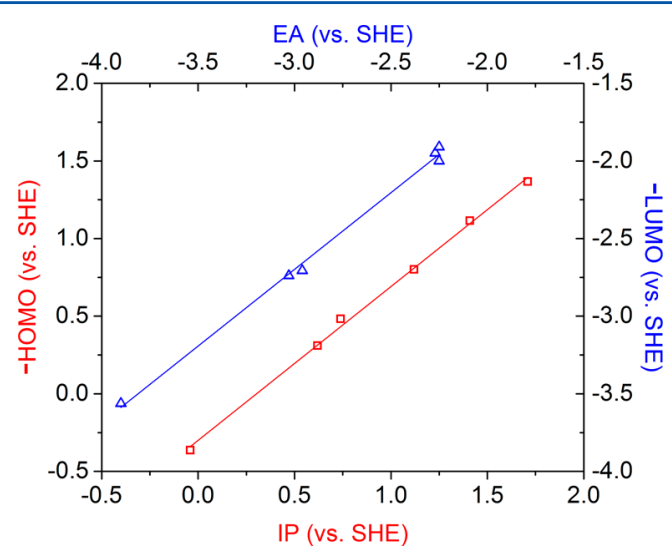

Figure 5. Comparison between IP and - HOMO, red open squares, and EA and -LUMO, open blue triangles, calculated with B3LYP and $\varepsilon_{\mathrm{r}} 2$.

and by extension with experimental IP/EA values. The difference between the orbital energies and potentials is an approximately constant small shift of a couple of tenths of volts. In line with what was previously observed by Schwenn and coworkers $^{67}$ in the case of small molecules, the reason again appears to be benign error cancellation. The underestimation of the vertical fundamental gap by the HOMO-LUMO energy difference is similar in size to a shift in IP and EA due to adiabatic and polarization effects not included in orbital energies, and both effects cancel each other. However, while we observe a decent fit between IP/EA and - HOMO/LUMO for the solid-state oligomers studied, the accuracy of unshifted orbital energies will be different for different dielectric constants (see the Supporting Information) and hence different environments, as well as, we expect, when using different density functionals or for the case of $\mathrm{PBC}$ calculations on solids.

\section{PERSPECTIVE}

Methodology. Consistently calculating the set of potentials associated with the charge carriers and exciton is rather a demanding application. Very likely other density functionals than B3LYP, for example optimally tuned range-separated density functionals, ${ }^{69,72,88}$ might yield more accurate values for the ionization potential or electron affinity of oligomers in the gas phase or in solution. However, to be useful in this context, use of such a functional should simultaneously allow for the calculations of the other potentials, including the excited state potentials, and hence the optical properties of a system, and the potentials of solution reactions, and all to a similar consistent standard. Additionally, while our dependence on error cancelation to predict decent solid-state values is unsatisfying from a theoretical point of view, it saves one from having to do calculations on extended models of the solid. If our estimate of the contribution of hybridization to the polarization energy for PPP is correct and a general feature of conjugated polymers, then calculations with functionals that give better gas phase values might require calculations on explicit stacks to obtain solid-state values or ad-hoc shifts. The latter is probably as conceptually unsatisfying as relying on error cancelation, while the former is, at least for polymers that are amorphous or poorly crystallized, difficult to achieve due to, as discussed in the Introduction, the lack of meaningful structural models. An additional complication with calculations on explicit stacks is the fact that the percentage of Hartree-Fock exchange included the density functional fixes the intermolecular dielectric screening inside the stack. ${ }^{89}$ As a result, the effective dielectric constant inside the stack might be different from its desired value and different from that used in the external continuum dielectric screening model.

We see the use of orbital energies to predominantly lie in fast screening of materials before calculating the potentials explicitly with $\triangle \mathrm{DFT}$ for promising materials. Even if the $-\mathrm{HOMO} /-$ LUMO values would be exactly identical to IP/EA, the fact that within the orbital formalism one cannot calculate the excited state potentials or estimate adiabatic or polarization effects makes it, in our opinion, at least for polymers, more of a high throughput screening tool than an in-depth analysis method.

Materials. Besides validating our computational methodology, the comparison between experimentally measured and computationally predicted potentials also gives us additional insight into polymeric materials and their ability to act as watersplitting photocatalysts. Specifically, the good match between the predicted and experimentally measured ionization potentials, as well as the specific experimental values of the ionization potentials and electron affinities for the polymers considered, supports our previous observation ${ }^{34,38}$ that conjugated polymers generally have a large thermodynamic driving force for proton reduction and a small(er) driving force, if at all, for water oxidation, i.e., that the electron affinities and ionization potentials of most polymers are relatively shallow. The experimental data are similarly in line with our prediction ${ }^{38}$ 
that the incorporation of heteroatoms should drive down the ionization potential of a polymer and improve the driving force for water oxidation if it results in a polymer with an electronpoor $\pi$-system, e.g., poly(pyridine), and reduce this driving force if the resulting polymer is electron-rich, i.e., poly(pyrrole) and poly(thiophene).

Perhaps even more interestingly, our estimate of the experimental adiabatic exciton-binding energy in substituted poly(thiophene) P3HT and poly(phenylvinylene) PPV of $\sim 0.7$ (e) $\mathrm{V}$, obtained above from the difference in the measured electron affinity ${ }^{58-60}$ and 2PPE intermediate state energy ${ }^{58,59}$ discussed above, as well as the fact that it is similar to our theoretical estimate, confirms our previous observation ${ }^{38}$ that excitons in conjugated polymers should be so strongly bound that they are unlikely to spontaneously fall apart in the bulk. The generation of free charge carriers, where free signifies that the charge carriers are not bound in the form of an exciton, thus has to be mediated by either a reduction of the adiabatic exciton-binding energy through induced dielectric screening by close contact with water or dissociation of the exciton at the polymer-water (or heteropolymer) interface. Calculations ${ }^{38}$ predict that the former indeed takes place but that the resulting adiabatic exciton-binding energies still will be larger than $k B^{*} T$ at room temperature. Free charge carrier generation thus appears mediated by interface dissociation of excitons, similar to exciton dissociation in organic photovoltaics on the donoracceptor interface. ${ }^{90}$

\section{CONCLUSIONS}

We have for a range of polymers relevant to photocatalysis compared the predictions of density functional theory for the redox potentials associated with charge carriers and excitons to those measured experimentally. We find for the ionization potentials of solid-state polymers in contact with vacuum, of the different classes of potentials available experimentally for conjugated polymers, the set measured under conditions which are the most similar to those during water splitting, a good fit between the values predicted using $\triangle \mathrm{B} 3 \mathrm{LYP}$ and those measured experimentally. Experimental data measured under similar conditions for the electron affinity and excited state ionization potential are much more limited, but we find a good fit to $\triangle B 3 L Y P$ in the latter case and a decent fit in the former. Overall, the comparison with experimental data gives good confidence in the use of $\triangle \mathrm{B} 3 \mathrm{LYP}$ to predict polymer potentials for solids and suggests that, if the effect of replacing the interface with vacuum by an interface with water is largely dielectric in nature, the here used approach should also give accurate predictions under water-splitting conditions.

In contrast to the case of solid-state polymers, the $\triangle \mathrm{B} 3 \mathrm{LYP}$ predicted ionization potentials for oligomers of $p$-phenylene in the gas phase and solutions and oligomers of fluorene are off by $0.5-0.6 \mathrm{~V}$ with respect to experiment. A combination of this observation and comparison of experimental and theoretical estimates of the polarization energy suggests that the consistently good fit for solid polymers may be the result of benign error cancellation. We lack similar data for the electron affinity and excited state potentials, but it stands to reason that the decent description of these potentials is similarly the result of error cancelation.

Besides validating our computational approach, the comparison between experimental and computational results, among other things, also confirms our previous prediction that generally excitons in conjugated polymers are so strongly bound that they do not spontaneously fall apart in the bulk. The generation of free charge carriers instead thus must be mediated by the dissociation of excitons on the polymersolution interface or polymer-polymer interface in heterogeneous materials.

\section{ASSOCIATED CONTENT}

\section{Supporting Information}

The Supporting Information is available free of charge on the ACS Publications website at DOI: 10.1021/acs.jpcc.6b11133.

Tables of potentials for alternative $\varepsilon_{\mathrm{r}}$ values, tables of solid-state potentials vs vacuum, results with larger basis sets, and orbital approximation data for $\varepsilon_{\mathrm{r}} 80.1$ (PDF) xyz coordinates of relevant DFT optimized structures (ZIP)

\section{AUTHOR INFORMATION}

\section{Corresponding Author}

*E-mail: m.zwijnenburg@ucl.ac.uk.

ORCID

Martijn A. Zwijnenburg: 0000-0001-5291-2130

Notes

The authors declare no competing financial interest.

\section{ACKNOWLEDGMENTS}

We kindly acknowledge Prof. Dave Adams, Dr. Enrico Berardo, Prof. Richard Buchner, Dr. Cristina Butchosa, Prof. Andrew Cooper, Dr. Sam Chong, Dr. Warren Duffy, Prof. Furio Cora, Prof. Iain McCulloch, Prof. Chad Risko, Dr. Sebastian Sprick, and Lei Yuang for stimulating discussions. M.A.Z. acknowledges the UK Engineering and Physical Sciences Research Council (EPSRC) for a Career Acceleration Fellowship (Grant $\mathrm{EP} / \mathrm{I004424/1)}$, as well as additional funding (EP/N004884/ 1). Computational time on Archer, the UK's national highperformance computing service (via our membership of the UK's HPC Materials Chemistry Consortium, which is funded by EPSRC grant EP/L000202/1), and at the EPSRC National Centre for Computational Chemistry Software (NCCCS) is gratefully acknowledged.

\section{REFERENCES}

(1) Yanagida, S.; Kabumoto, A.; Mizumoto, K.; Pac, C.; Yoshino, K. Poly(Para-Phenylene)-Catalyzed Photoreduction of Water to Hydrogen. J. Chem. Soc., Chem. Commun. 1985, 474-475.

(2) Shibata, T.; Kabumoto, A.; Shiragami, T.; Ishitani, O.; Pac, C.; Yanagida, S. Novel Visible-Light-Driven Photocatalyst - Poly(ParaPhenylene)-Catalyzed Photoreductions of Water, Carbonyl-Compounds, and Olefins. J. Phys. Chem. 1990, 94, 2068-2076.

(3) Matsuoka, S.; Fujii, H.; Pac, C.; Yanagida, S. Photocatalysis of Oligo(Para-Phenylene) Leading to Reductive Formation of Hydrogen and Ethanol from Triethylamine in Aqueous Organic-Solvent. Chem. Lett. 1990, 19, 1501-1502.

(4) Matsuoka, S.; Kohzuki, T.; Pac, C.; Ishida, A.; Takamuku, S.; Kusaba, M.; Nakashima, N.; Yanagida, S. Photocatalysis of Oligo(ParaPhenylenes) - Photochemical Reduction of Carbon-Dioxide with Triethylamine. J. Phys. Chem. 1992, 96, 4437-4442.

(5) Schwab, M. G.; Hamburger, M.; Feng, X. L.; Shu, J.; Spiess, H. W.; Wang, X. C.; Antonietti, M.; Mullen, K. Photocatalytic Hydrogen Evolution through Fully Conjugated Poly(Azomethine) Networks. Chem. Commun. 2010, 46, 8932-8934.

(6) Zhang, Z. Z.; Long, J. L.; Yang, L. F.; Chen, W. K.; Dai, W. X.; Fu, X. Z.; Wang, X. X. Organic Semiconductor for Artificial Photosynthesis: Water Splitting into Hydrogen by a Bioinspired 
$\mathrm{C}_{3} \mathrm{~N}_{3} \mathrm{~S}_{3}$ Polymer under Visible Light Irradiation. Chem. Sci. 2011, 2, $1826-1830$

(7) Chu, S.; Wang, Y.; Guo, Y.; Zhou, P.; Yu, H.; Luo, L. L.; Kong, F.; Zou, Z. G. Facile Green Synthesis of Crystalline Polyimide Photocatalyst for Hydrogen Generation from Water. J. Mater. Chem. 2012, 22, 15519-15521.

(8) Sprick, R. S.; Jiang, J. X.; Bonillo, B.; Ren, S. J.; Ratvijitvech, T.; Guiglion, P.; Zwijnenburg, M. A.; Adams, D. J.; Cooper, A. I. Tunable Organic Photocatalysts for Visible-Light-Driven Hydrogen Evolution. J. Am. Chem. Soc. 2015, 137, 3265-3270.

(9) Bornoz, P.; Prevot, M. S.; Yu, X. Y.; Guijarro, N.; Sivula, K. Direct Light-Driven Water Oxidation by a Ladder-Type Conjugated Polymer Photoanode. J. Am. Chem. Soc. 2015, 137, 15338-15341.

(10) Schwinghammer, K.; Hug, S.; Mesch, M. B.; Senker, J.; Lotsch, B. V. Phenyl-Triazine Oligomers for Light-Driven Hydrogen Evolution. Energy Environ. Sci. 2015, 8, 3345-3353.

(11) Sprick, R. S.; Bonillo, B.; Clowes, R.; Guiglion, P.; Brownbill, N. J.; Slater, B. J.; Blanc, F.; Zwijnenburg, M. A.; Adams, D. J.; Cooper, A. I. Visible-Light-Driven Hydrogen Evolution Using Planarized Conjugated Polymer Photocatalysts. Angew. Chem. 2016, 128, 1824-1828.

(12) Yang, C.; Ma, B. C.; Zhang, L. Z.; Lin, S.; Ghasimi, S.; Landfester, K.; Zhang, K. A. I.; Wang, X. C. Molecular Engineering of Conjugated Polybenzothiadiazoles for Enhanced Hydrogen Production by Photosynthesis. Angew. Chem., Int. Ed. 2016, 55, 9202-9206.

(13) Li, L. W.; Cai, Z. X.; Wu, Q. H.; Lo, W. Y.; Zhang, N.; Chen, L. $\mathrm{X}$.; Yu, L. P. Rational Design of Porous Conjugated Polymers and Roles of Residual Palladium for Photocatalytic Hydrogen Production. J. Am. Chem. Soc. 2016, 138, 7681-7686.

(14) Vyas, V. S.; Lau, V. W. H.; Lotsch, B. V. Soft Photocatalysis: Organic Polymers for Solar Fuel Productions. Chem. Mater. 2016, 28, 5191-5204.

(15) Zhang, G.; Lan, Z.-A.; Wang, X. Organic Conjugated Semiconductors for Photocatalytic Hydrogen Evolution with Visible Light. Angew. Chem., Int. Ed. 2016, 55, 15712-15727.

(16) Wang, X. C.; Maeda, K.; Thomas, A.; Takanabe, K.; Xin, G.; Carlsson, J. M.; Domen, K.; Antonietti, M. A Metal-Free Polymeric Photocatalyst for Hydrogen Production from Water under Visible Light. Nat. Mater. 2009, 8, 76-80.

(17) Wang, X. C.; Maeda, K.; Chen, X. F.; Takanabe, K.; Domen, K.; Hou, Y. D.; Fu, X. Z.; Antonietti, M. Polymer Semiconductors for Artificial Photosynthesis: Hydrogen Evolution by Mesoporous Graphitic Carbon Nitride with Visible Light. J. Am. Chem. Soc. 2009, 131, $1680-1681$

(18) Maeda, K.; Wang, X. C.; Nishihara, Y.; Lu, D. L.; Antonietti, M.; Domen, K. Photocatalytic Activities of Graphitic Carbon Nitride Powder for Water Reduction and Oxidation under Visible Light. J. Phys. Chem. C 2009, 113, 4940-4947.

(19) Zhang, J. S.; Chen, X. F.; Takanabe, K.; Maeda, K.; Domen, K.; Epping, J. D.; Fu, X. Z.; Antonietti, M.; Wang, X. C. Synthesis of a Carbon Nitride Structure for Visible-Light Catalysis by Copolymerization. Angew. Chem., Int. Ed. 2010, 49, 441-444.

(20) Zhang, G. G.; Lan, Z. A.; Lin, L. H.; Lin, S.; Wang, X. C. Overall Water Splitting by $\mathrm{Pt} / \mathrm{G}-\mathrm{C}_{3} \mathrm{~N}_{4}$ Photocatalysts without Using Sacrificial Agents. Chem. Sci. 2016, 7, 3062-3066.

(21) Sui, Y.; Liu, J. H.; Zhang, Y. W.; Tian, X. K.; Chen, W. Dispersed Conductive Polymer Nanoparticles on Graphitic Carbon Nitride for Enhanced Solar-Driven Hydrogen Evolution from Pure Water. Nanoscale 2013, 5, 9150-9155.

(22) Schwinghammer, K.; Tuffy, B.; Mesch, M. B.; Wirnhier, E.; Martineau, C.; Taulelle, F.; Schnick, W.; Senker, J.; Lotsch, B. V. Triazine-Based Carbon Nitrides for Visible-Light-Driven Hydrogen Evolution. Angew. Chem., Int. Ed. 2013, 52, 2435-2439.

(23) Martin, D. J.; Qiu, K. P.; Shevlin, S. A.; Handoko, A. D.; Chen, X. W.; Guo, Z. X.; Tang, J. W. Highly Efficient Photocatalytic $\mathrm{H}_{2}$ Evolution from Water Using Visible Light and Structure-Controlled Graphitic Carbon Nitride. Angew. Chem., Int. Ed. 2014, 53, 92409245.

(24) Schwinghammer, K.; Mesch, M. B.; Duppel, V.; Ziegler, C.; Senker, J.; Lotsch, B. V. Crystalline Carbon Nitride Nanosheets for
Improved Visible-Light Hydrogen Evolution. J. Am. Chem. Soc. 2014, 136, 1730-1733.

(25) Liu, J.; Liu, Y.; Liu, N. Y.; Han, Y. Z.; Zhang, X.; Huang, H.; Lifshitz, Y.; Lee, S. T.; Zhong, J.; Kang, Z. H. Metal-Free Efficient Photocatalyst for Stable Visible Water Splitting Via a Two-Electron Pathway. Science 2015, 347, 970-974.

(26) Lau, V. W. H.; Mesch, M. B.; Duppel, V.; Blum, V.; Senker, J.; Lotsch, B. V. Low-Molecular-Weight Carbon Nitrides for Solar Hydrogen Evolution. J. Am. Chem. Soc. 2015, 137, 1064-1072.

(27) Lau, V. W. H.; Moudrakovski, I.; Botari, T.; Weinberger, S.; Mesch, M. B.; Duppel, V.; Senker, J.; Blum, V.; Lotsch, B. V. Rational Design of Carbon Nitride Photocatalysts by Identification of Cyanamide Defects as Catalytically Relevant Sites. Nat. Commun. 2016, 7, 12165.

(28) Yeh, T. F.; Syu, J. M.; Cheng, C.; Chang, T. H.; Teng, H. S. Graphite Oxide as a Photocatalyst for Hydrogen Production from Water. Adv. Funct. Mater. 2010, 20, 2255-2262.

(29) Yeh, T. F.; Teng, C. Y.; Chen, S. J.; Teng, H. S. Nitrogen-Doped Graphene Oxide Quantum Dots as Photocatalysts for Overall WaterSplitting under Visible Light Illumination. Adv. Mater. 2014, 26, 3297-3303.

(30) Tsai, E. W.; Basak, S.; Ruiz, J. P.; Reynolds, J. R.; Rajeshwar, K. Electrochemistry of Some Beta-Substituted Polythiophenes - AnodicOxidation, Electrochromism, and Electrochemical Deactivation. J. Electrochem. Soc. 1989, 136, 3683-3689.

(31) Novak, P.; Rasch, B.; Vielstich, W. Overoxidation of Polypyrrole in Propylene Carbonate - an Insitu FTIR Study. J. Electrochem. Soc. 1991, 138, 3300-3304.

(32) Zotti, G.; Schiavon, G.; Zecchin, S. Irreversible-Processes in the Electrochemical Reduction of Polythiophenes - Chemical Modifications of the Polymer and Charge-Trapping Phenomena. Synth. Met. 1995, 72, 275-281.

(33) Vyas, R. N.; Wang, B. Electrochemical Analysis of Conducting Polymer Thin Films. Int. J. Mol. Sci. 2010, 11, 1956-1972.

(34) Guiglion, P.; Butchosa, C.; Zwijnenburg, M. A. Polymeric Watersplitting Photocatalysts; a Computational Perspective on the Water Oxidation Conundrum. J. Mater. Chem. A 2014, 2, 1199612004.

(35) Butchosa, C.; Guiglion, P.; Zwijnenburg, M. A. Carbon Nitride Photocatalysts for Water Splitting: A Computational Perspective. J. Phys. Chem. C 2014, 118, 24833-24842.

(36) Berardo, E.; Zwijnenburg, M. A. Modeling the Water Splitting Activity of a Tio2 Rutile Nanoparticle. J. Phys. Chem. C 2015, 119, 13384-13393.

(37) Guiglion, P.; Berardo, E.; Butchosa, C.; Wobbe, M. C. C.; Zwijnenburg, M. A. Modelling Materials for Solar Fuel Synthesis by Artificial Photosynthesis; Predicting the Optical, Electronic and Redox Properties of Photocatalysts. J. Phys.: Condens. Matter 2016, 28, 074001.

(38) Guiglion, P.; Butchosa, C.; Zwijnenburg, M. A. Polymer Photocatalysts for Water Splitting: Insights from Computational Modeling. Macromol. Chem. Phys. 2016, 217, 344-353.

(39) Toroker, M. C.; Kanan, D. K.; Alidoust, N.; Isseroff, L. Y.; Liao, P. L.; Carter, E. A. First Principles Scheme to Evaluate Band Edge Positions in Potential Transition Metal Oxide Photocatalysts and Photoelectrodes. Phys. Chem. Chem. Phys. 2011, 13, 16644-16654.

(40) Castelli, I. E.; Olsen, T.; Datta, S.; Landis, D. D.; Dahl, S.; Thygesen, K. S.; Jacobsen, K. W. Computational Screening of Perovskite Metal Oxides for Optimal Solar Light Capture. Energy Environ. Sci. 2012, 5, 5814-5819.

(41) Persson, K. A.; Waldwick, B.; Lazic, P.; Ceder, G. Prediction of Solid-Aqueous Equilibria: Scheme to Combine First-Principles Calculations of Solids with Experimental Aqueous States. Phys. Rev. B: Condens. Matter Mater. Phys. 2012, 85, 235438.

(42) Zhuang, H. L. L.; Hennig, R. G. Single-Layer Group-Iii Monochalcogenide Photocatalysts for Water Splitting. Chem. Mater. 2013, 25, 3232-3238.

(43) Stevanovic, V.; Lany, S.; Ginley, D. S.; Tumas, W.; Zunger, A. Assessing Capability of Semiconductors to Split Water Using 
Ionization Potentials and Electron Affinities Only. Phys. Chem. Chem. Phys. 2014, 16, 3706-3714.

(44) Jiang, X.; Wang, P.; Zhao, J. J. 2d Covalent Triazine Framework: A New Class of Organic Photocatalyst for Water Splitting. J. Mater. Chem. A 2015, 3, 7750-7758.

(45) Buckeridge, J.; Butler, K. T.; Catlow, C. R. A.; Logsdail, A. J.; Scanlon, D. O.; Shevlin, S. A.; Woodley, S. M.; Sokol, A. A.; Walsh, A. Polymorph Engineering of Tio2: Demonstrating How Absolute Reference Potentials Are Determined by Local Coordination. Chem. Mater. 2015, 27, 3844-3851.

(46) Tomasi, J.; Mennucci, B.; Cammi, R. Quantum Mechanical Continuum Solvation Models. Chem. Rev. 2005, 105, 2999-3093.

(47) Baerends, E. J.; Gritsenko, O. V.; van Meer, R. The Kohn-Sham Gap, the Fundamental Gap and the Optical Gap: The Physical Meaning of Occupied and Virtual Kohn-Sham Orbital Energies. Phys. Chem. Chem. Phys. 2013, 15, 16408-16425.

(48) Seki, K.; Karlsson, U. O.; Engelhardt, R.; Koch, E. E.; Schmidt, W. Intramolecular Band Mapping of Poly(Para-Phenylene) Via Uv Photoelectron-Spectroscopy of Finite Polyphenyls. Chem. Phys. 1984, 91, 459-470.

(49) Banerjee, M.; Shukla, R.; Rathore, R. Synthesis, Optical, and Electronic Properties of Soluble Poly-P-Phenylene Oligomers as Models for Molecular Wires. J. Am. Chem. Soc. 2009, 131, 1780-1786.

(50) Chi, C. Y.; Wegner, G. Chain-Length Dependence of the Electrochemical Properties of Conjugated Oligofluorenes. Macromol. Rapid Commun. 2005, 26, 1532-1537.

(51) Nagashima, U.; Fujimoto, H.; Inokuchi, H.; Seki, K. Electronic and Geometric Structures of Oligothiophenes. J. Mol. Struct. 1989, 197, 265-289.

(52) Seki, K.; Asada, S.; Mori, T.; Inokuchi, H.; Murase, I.; Ohnishi, T.; Noguchi, T. Uv Photoemission Spectroscopy of Poly(P-Phenylene Vinylene) (PPV). Solid State Commun. 1990, 74, 677-680.

(53) Fujimoto, H.; Nagashima, U.; Inokuchi, H.; Seki, K.; Nakahara, N.; Nakayama, J.; Hoshino, M.; Fukuda, K. Electronic and Geometric Structures of Oligothiophenes Studied by Ups and Mndo - $\pi$-Band Evolution and Effect of Disorder. Phys. Scr. 1990, 41, 105-109.

(54) Hino, S.; Iwasaki, K.; Tatematsu, H.; Matsumoto, K. Photoelectron-Spectra of Polypyrrole - the Effect of the Ambient Atmosphere to the Spectra. Bull. Chem. Soc. Jpn. 1990, 63, 2199-2205.

(55) Miyamae, T.; Yoshimura, D.; Ishii, H.; Ouchi, Y.; Seki, K.; Miyazaki, T.; Koike, T.; Yamamoto, T. Ultraviolet PhotoelectronSpectroscopy of Poly(Pyridine-2,5-Diyl), Poly(2,2'-Bipyridine-5,5'Diyl), and Their K-Doped States. J. Chem. Phys. 1995, 103, 27382744.

(56) Liao, L. S.; Fung, M. K.; Lee, C. S.; Lee, S. T.; Inbasekaran, M.; Woo, E. P.; Wu, W. W. Electronic Structure and Energy Band Gap of Poly (9,9-Dioctylfluorene) Investigated by Photoelectron Spectroscopy. Appl. Phys. Lett. 2000, 76, 3582-3584.

(57) Hwang, J.; Kim, E. G.; Liu, J.; Bredas, J. L.; Duggal, A.; Kahn, A. Photoelectron Spectroscopic Study of the Electronic Band Structure of Polyfluorene and Fluorene-Arylamine Copolymers at Interfaces. J. Phys. Chem. C 2007, 111, 1378-1384.

(58) Sohn, Y.; Stuckless, J. T. Bimolecular Recombination Kinetics and Interfacial Electronic Structures of Poly 2-Methoxy-5-(2-EthylHexyloxy)-P-Phenylene Vinylene on Gold Studied Using Two-Photon Photoemission Spectroscopy. J. Chem. Phys. 2007, 126, 174901.

(59) Sohn, Y.; Stuckless, J. T. Characteristics of Photoexcitations and Interfacial Energy Levels of Regioregular Poly(3-Hexythiophene-2,5Diyl) on Gold. ChemPhysChem 2007, 8, 1937-1942.

(60) Kanai, K.; Miyazaki, T.; Suzuki, H.; Inaba, M.; Ouchi, Y.; Seki, K. Effect of Annealing on the Electronic Structure of Poly(3Hexylthiophene) Thin Film. Phys. Chem. Chem. Phys. 2010, 12, 273-282.

(61) Baik, M. H.; Friesner, R. A. Computing Redox Potentials in Solution: Density Functional Theory as a Tool for Rational Design of Redox Agents. J. Phys. Chem. A 2002, 106, 7407-7412.

(62) Uudsemaa, M.; Tamm, T. Density-Functional Theory Calculations of Aqueous Redox Potentials of Fourth-Period Transition Metals. J. Phys. Chem. A 2003, 107, 9997-10003.
(63) Shimodaira, Y.; Miura, T.; Kudo, A.; Kobayashi, H. Dft Method Estimation of Standard Redox Potential of Metal Ions and Metal Complexes. J. Chem. Theory Comput. 2007, 3, 789-795.

(64) Roy, L. E.; Jakubikova, E.; Guthrie, M. G.; Batista, E. R Calculation of One-Electron Redox Potentials Revisited. Is It Possible to Calculate Accurate Potentials with Density Functional Methods? J. Phys. Chem. A 2009, 113, 6745-6750.

(65) Nayak, P. K.; Periasamy, N. Calculation of Ionization Potential of Amorphous Organic Thin-Films Using Solvation Model and DFT. Org. Electron. 2009, 10, 532-535.

(66) Nayak, P. K.; Periasamy, N. Calculation of Electron Affinity, Ionization Potential, Transport. Gap, Optical Band Gap and Exciton Binding Energy of Organic Solids Using 'Solvation' Model and DFT. Org. Electron. 2009, 10, 1396-1400.

(67) Schwenn, P. E.; Burn, P. L.; Powell, B. J. Calculation of Solid State Molecular Ionisation Energies and Electron Affinities for Organic Semiconductors. Org. Electron. 2011, 12, 394-403.

(68) Hughes, T. F.; Friesner, R. A. Development of Accurate Dft Methods for Computing Redox Potentials of Transition Metal Complexes: Results for Model Complexes and Application to Cytochrome P450. J. Chem. Theory Comput. 2012, 8, 442-459.

(69) Tamblyn, I.; Refaely-Abramson, S.; Neaton, J. B.; Kronik, L. Simultaneous Determination of Structures, Vibrations, and Frontier Orbital Energies from a Self-Consistent Range-Separated Hybrid Functional. J. Phys. Chem. Lett. 2014, 5, 2734-2741.

(70) Phillips, H.; Zheng, Z. L.; Geva, E.; Dunietz, B. D. Orbital Gap Predictions for Rational Design of Organic Photovoltaic Materials. Org. Electron. 2014, 15, 1509-1520.

(71) Richard, R. M.; Marshall, M. S.; Dolgounitcheva, O.; Ortiz, J. V.; Bredas, J. L.; Marom, N.; Sherrill, C. D. Accurate Ionization Potentials and Electron Affinities of Acceptor Molecules I. Reference Data at the $\operatorname{CCSD}(\mathrm{T})$ Complete Basis Set Limit. J. Chem. Theory Comput. 2016, $12,595-604$.

(72) Gallandi, L.; Marom, N.; Rinke, P.; Korzdorfer, T. Accurate Ionization Potentials and Electron Affinities of Acceptor Molecules II: Non-Empirically Tuned Long-Range Corrected Hybrid Functionals. J. Chem. Theory Comput. 2016, 12, 605-614.

(73) Rangel, T.; Hamed, S. M.; Bruneval, F.; Neaton, J. B. Evaluating the Gw Approximation with $\operatorname{CCSD}(\mathrm{T})$ for Charged Excitations across the Oligoacenes. J. Chem. Theory Comput. 2016, 12, 2834-2842.

(74) Becke, A. D. Density-Functional Thermochemistry 0.3. The Role of Exact Exchange. J. Chem. Phys. 1993, 98, 5648-5652.

(75) Stephens, P. J.; Devlin, F. J.; Chabalowski, C. F.; Frisch, M. J. $\mathrm{Ab}$-Initio Calculation of Vibrational Absorption and CircularDichroism Spectra Using Density-Functional Force-Fields. J. Phys. Chem. 1994, 98, 11623-11627.

(76) Klamt, A.; Schuurmann, G. Cosmo - a New Approach to Dielectric Screening in Solvents with Explicit Expressions for the Screening Energy and Its Gradient. J. Chem. Soc., Perkin Trans. 2 1993, 799-805.

(77) Hirata, S.; Head-Gordon, M. Time-Dependent Density Functional Theory within the Tamm-Dancoff Approximation. Chem. Phys. Lett. 1999, 314, 291-299.

(78) Furche, F.; Ahlrichs, R.; Hattig, C.; Klopper, W.; Sierka, M.; Weigend, F. Turbomole. Wiley Interdiscip. Rev. Comput. Mol. Sci. 2014, 4, 91-100.

(79) Ahlrichs, R.; Bar, M.; Haser, M.; Horn, H.; Kolmel, C. Electronic-Structure Calculations on Workstation Computers - the Program System Turbomole. Chem. Phys. Lett. 1989, 162, 165-169.

(80) Schafer, A.; Horn, H.; Ahlrichs, R. Fully Optimized Contracted Gaussian-Basis Sets for Atoms Li to Kr. J. Chem. Phys. 1992, 97, 25712577.

(81) Isse, A. A.; Gennaro, A. Absolute Potential of the Standard Hydrogen Electrode and the Problem of Interconversion of Potentials in Different Solvents. J. Phys. Chem. B 2010, 114, 7894-7899.

(82) Trasatti, S. The Absolute Electrode Potential - an Explanatory Note (Recommendations 1986). Pure Appl. Chem. 1986, 58, 955-966. 
(83) Sato, N.; Seki, K.; Inokuchi, H. Polarization Energies of OrganicSolids Determined by Ultraviolet Photoelectron-Spectroscopy. J. Chem. Soc., Faraday Trans. 2 1981, 77, 1621-1633.

(84) Inokuchi, H.; Seki, K.; Sato, N. Uv Photoelectron-Spectroscopy of Organic Molecular Materials. Phys. Scr. 1987, T17, 93-103.

(85) Gestblom, B.; Songstad, J. Solvent Properties of Dichloromethane 0.6. Dielectric-Properties of Electrolytes in Dichloromethane. Acta Chem. Scand. 1987, 41, 396-409.

(86) Tsiper, E. V.; Soos, Z. G.; Gao, W.; Kahn, A. Electronic Polarization at Surfaces and Thin Films of Organic Molecular Crystals: Ptcda. Chem. Phys. Lett. 2002, 360, 47-52.

(87) Ryno, S. M.; Risko, C.; Bredas, J. L. Impact of Molecular Packing on Electronic Polarization in Organic Crystals: The Case of Pentacene Vs Tips-Pentacene. J. Am. Chem. Soc. 2014, 136, 64216427.

(88) Stein, T.; Eisenberg, H.; Kronik, L.; Baer, R. Fundamental Gaps in Finite Systems from Eigenvalues of a Generalized Kohn-Sham Method. Phys. Rev. Lett. 2010, 105, 266802.

(89) Refaely-Abramson, S.; Sharifzadeh, S.; Jain, M.; Baer, R.; Neaton, J. B.; Kronik, L. Gap Renormalization of Molecular Crystals from Density-Functional Theory. Phys. Rev. B: Condens. Matter Mater. Phys. 2013, 88, 081204.

(90) Few, S.; Frost, J. M.; Nelson, J. Models of Charge Pair Generation in Organic Solar Cells. Phys. Chem. Chem. Phys. 2015, 17, $2311-2325$. 